\title{
LE GITE TITANIFERE DE PEDRA COMPRIDA (MUNICIPE DE CAMPO ALEGRE DE LOURDES - ETAT DE BAHIA)
}

\author{
J. P. CASSEDANNE*, J. O. CASSEDANNE* et R. MARANHÃO**
}

\begin{abstract}
This paper describes an ilmenite-hematite ore deposit. This is an elongated lens-shaped bed conforms with the regional Upper Precambrian gneiss. The deposit lies in the North of the State of Bahia, in the Pedra Comprida area, Campo Alegre de Lourdes township.

The ore is made of large magnesium ilmenite crystals with variable corrosion, embedded in one homogeneous and very fine mass of hematite with exsolved common ilmenite. The ore is very few brecciated and invaded by gangue minerals (mica and feldspars). Magnetite and rutile are rare. Sulfides are lacking. Goethite and leucoxene are poorly represented.

The magnesium ilmenite, very rare mineral in this ore deposit type, is examined by diffractometric, microscopic and chemical processes.

Genetically, the ore deposit of Campo Alegre de Lourdes is magmatic, but exceptionnal by the magnesium ilmenite and the lack of anorthositic complex in the vicinity.
\end{abstract}

RESUMO Esse trabalho descreve uma jazida de ilmenita e hematita, em forma de lente alongada, concordante com os gnaisses encaixantes atribuíveis ao Pré-Cambriano Superior. Ela está situada no norte do Estado da Bahia, no Município de Campo Alegre de Lourdes, na localidade de Pedra Comprida.

O minério é constituído por grandes cristais de ilmenita magnesiana, mais ou menos corroídos, envolvidos por um cimento bastante homogêneo e fino de hematita com exsolução de ilmenita comum. O conjunto se apresenta pouco deslocado e invadido pela ganga (mica e feldspato). A magnetita é rara assim como o rutílio. Não há sulfetos. Goethita e leucoxênio são pouco abundantes.

$O$ estudo difratométrico da ilmenita magnesiana, mineral excepcional nesse tipo de jazida, é dado, assim como suas características mineralógicas e químicas.

Do ponto de vista da classificação, a jazida de Campo Alegre é de origem magmática, porém de tipo excepcional: presença de ilmenita magnesiana e ausência de série anortosítica nas vizinhanças.

INTRODUCTION Moyens d'accés Comme on ne connait pas d'indices ou de gisements d'ilménite en roche au Brésil, nous nous proposons de décrire ci-dessous les affleurements titanifères existant près du hameau de Pedra Comprida, dont l'importance permet de présager le probable intérêt économique, raison pour laquelle nous nous y référerons comme gîte dans le cours du présent travail.

Ces affleurements sont situés au voisinage de Campo Alegre de Lourdes, dans l'extrême Nord de l'Etat de Bahia, au voisinage de la frontière de celui du Piauí, à 105 km environ à l'WSW de Remanso et à $150 \mathrm{~km}$ au NNW de Xique-Xique.

L'accès se fait généralement par Remanso (altitude $390 \mathrm{~m}$ ), relié par une bonne route de terre encaillassée à Petrolina $(202 \mathrm{~km}$, dont 72 goudronnés jusqu'à Casa Nova).

*CNPq e Instituto de Geociências, Universidade Federal do Rio de Janeiro, Cidade Universitária, Ilha do Fundão, Rio de Janeiro, RJ

**Instituto de Geociências, Universidade Federal de Pernambuco, Cidade Universitária, Engenho do Meio, Recife, PE 
A partir de Remanso une piste sableuse serpente jusqu'à Campo Alegre (480 m) durant $115 \mathrm{~km}$, via Angico à $80 \mathrm{~km}$ de la première ville. Un chemin de terre permet ensuite d'atteindre facilement les affleurements en $10 \mathrm{~km}$ jusqu'à la Pedra Comprida. Un autre se dirige vers Fósforo et recoupe le Morro Velho. Enfin, un sentier suit sur plus de $2 \mathrm{~km}$ la base du Morro da Pedra Comprida, pendant qu'un autre, carrossable, rejoint la fazenda da Pedra Comprida.

Contexte regional 1. GENERALITES La région voisine de la minéralisation, très plane, est uniquement constituée par des terrains métamorphiques formant une vaste plaine au sol sableux d'où émergent de rares élévations aux pentes douces.

Les affleurements dénudés, en larges plaques ou blocs arrondis, sont fréquents, séparés par des étendues sableuses où divaguent des rios généralement à sec.

On remarque des croûtes latéritiques sur les reliefs, au voisinage de la cote 510 .

La caatinga couvre cette zone peu habitée où l'eau est rare.

2. TOPOGRAPHIE Une série de collines NNE-SSW au Nord, devenant subméridiennes au Sud, renferment les minéralisations présentement étudiées. On y distingue: le Morro Vermelho au Nord, le Morro Velho au SE et le Morro da Pedra Comprida au Sud, séparé du précédent par la vallée évasée du Riacho da Lagoa de Dentro (Carte 1).

Le Morro da Pedra Comprida se subdivise lui-même en deux élévations, dont la plus occidentale est connue sous le nom de Ponta da Serra. La Lagoa de Dentro, occupant une étroite dépression, sépare ces deux reliefs, que le Morro da Carlota et celui du Teiú prolongent vers le SW.

3. GEOLOGIE O. Barbosa (1965), le premier auteur à décrire la géologie régionale, a distingué:

Le groupe Caraiba d'âge supérieur à 2200 m.a. essentiellement constitué de gneiss migmatitiques associés à des leptinites, amphibolites et micaschistes dont le métamorphisme est du faciès almandin-amphibole (M. A. L. Bruni et al., 1974).

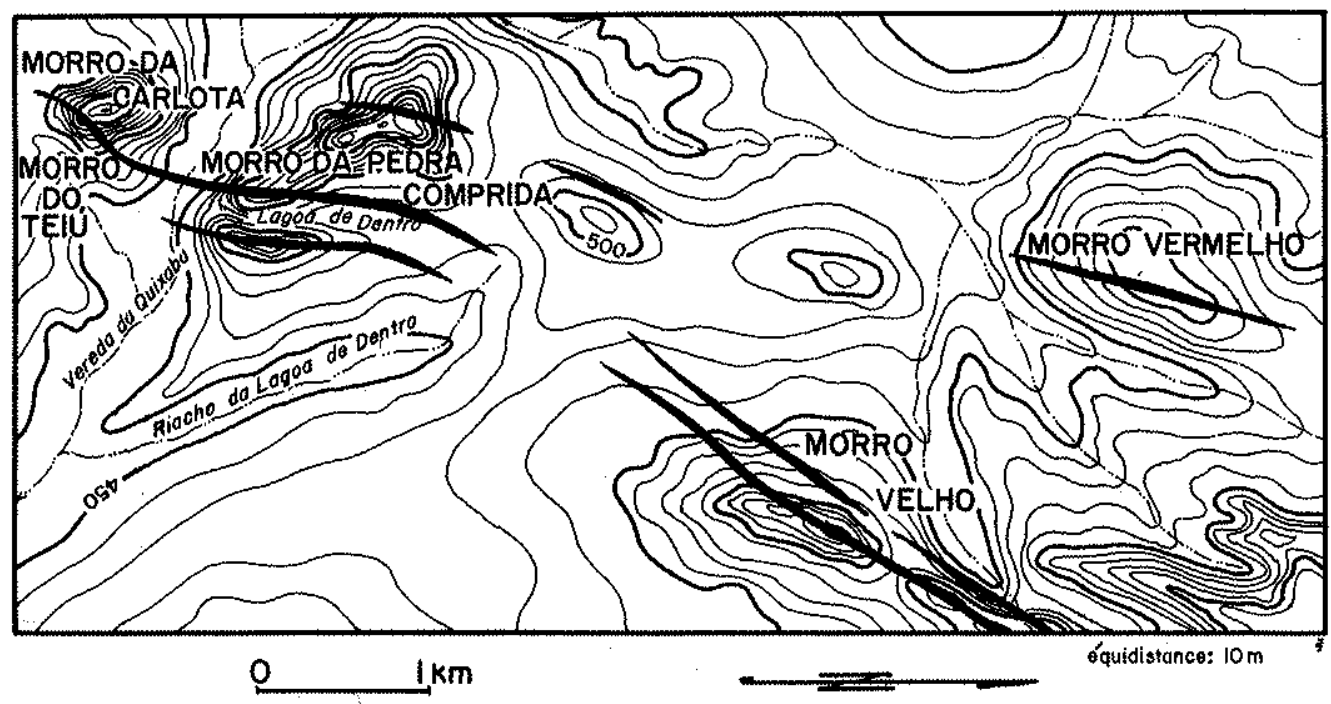

Carte 1 - Carte des affleurements de minerai 
Le groupe Colomi reposant en discordance sur le précédent et affleurant en taches isolées. Ses principales roches sont des quartzites, des dolomies, des schistes talqueux et à trémolite et des itabirites. Age: 1700-2 200 m.a.

Le groupe Salgueiro, également discordant sur les précédents et essentiellement constitué de micaschiste à deux micas avec quelques quartzites et calcaires lenticulaires, recoupés par des granites et des syénites. Age: 570-1 100 m.a.

Postérieurement M. Winge (1968) n'a, quant à lui, reconnu que deux unités lithologiques et stratigraphiques:

Un complexe ancien du Précambrien inférieur à moyen, formé de gneiss et de micaschistes métasomatisés en migmatites variées.
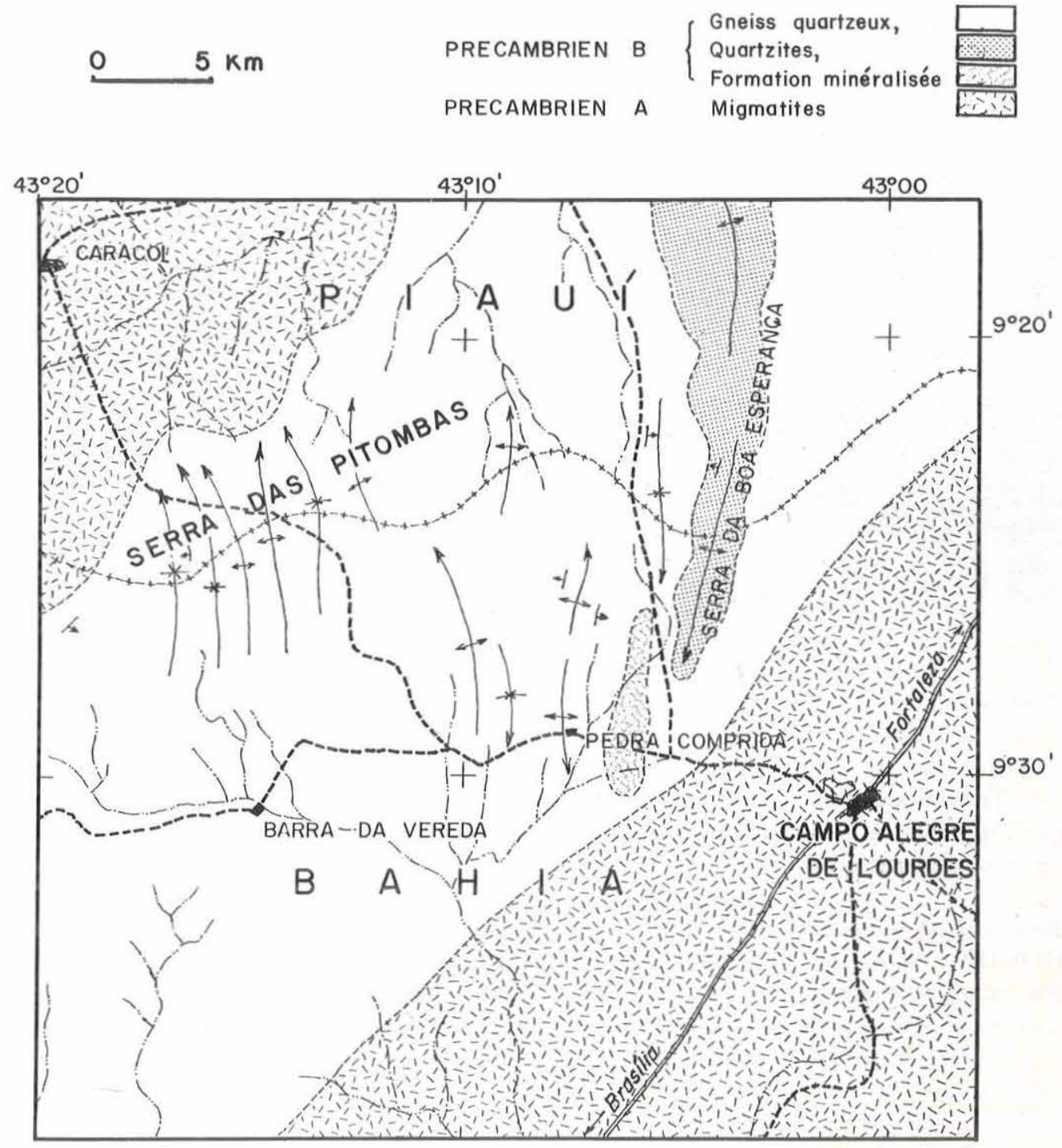

Carte 2 - Carte géologique régionale 
Le groupe Itacolomi du Précambrien supérieur, reposant en discordance importante sur le complexe précédent. Il est constitué de roches de plus bas degré de métamorphisme: phyllites, micaschistes et quartzites associés à des itabirites.

Enfin A. L. Caldasso et al. (1973) durant un récent lever régional ont distingué (Carte 2):

Le Précambrien A représenté par des migmatites homogènes (principalement anatexites) auxquelles s'associent localement des gneiss grossiers et des leptinites.

Cette unité forme l'essentiel de la région étudiée, constituant le soubassement de vastes plaines sableuses aux affleurements peu fréquents et que surplombent les reliefs du Précambrien B.

Le Précambrien $B$, qui constitue les grandes serras da Boa Esperança, da Pitombeira et do Estreito, affleure au voisinage de Campo Alegre sous la forme d'une bande d'environ $25 \mathrm{~km}$ de large. Il correspond au groupe Colomi de O. Barbosa et Itacolomi de M. Winge (1968).

La roche prédominante est un gneiss quartzeux encaissant des quartzites, conglomérats, micaschistes, calcaires cristallins et des lentilles de minerai de fer. Ges dernières se présentent em crêtes allongées dominant les plaines voisines (Fig. 1).

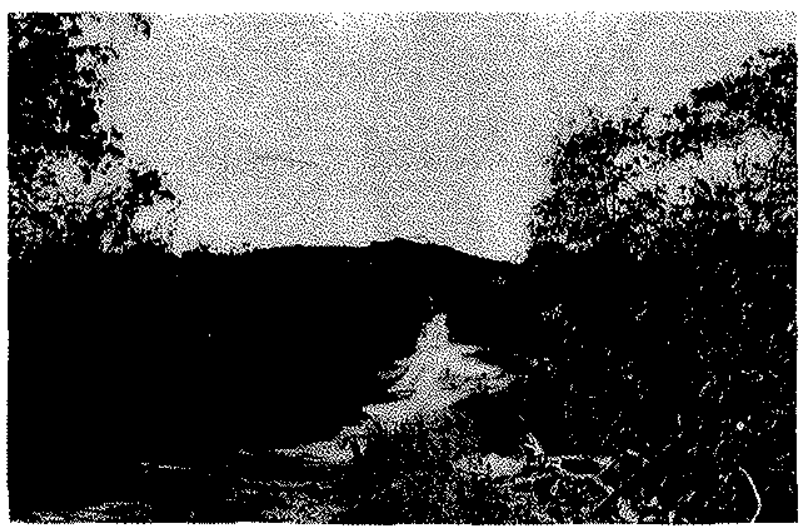

Figure 1 - Le Morro da Pedra Comprida, à l'horizon, domine une région basse que traverse la piste d'accès

Le gneiss est clair, à grain moyen à grossier, essentiellement formé de quartz et de feldspath, avec biotite subordonnée.

Le Précambrien C, enfin, est peu représenté, d'âge probablement Eo-Cambrien. Il est constitué par un métaconglomérat à ciment calcaire.

Tectoniquement, à l'échelle régionale, les roches du Précambrien $\mathbf{B}$ se caractérisent par une série de plis serrés s'ennoyant vers le Nord comme on peut l'observer dans les terminaisons périclinales des serras do Estreito et da Pitombeira.

Description succincte de la zone mineralisee 1. GEOLOGIE REGIONALE ROCHE ENCAISSANTE La zone minéralisée, objet de cette étude, ne présente que des roches du Précambrien $\mathbf{B}$ qui peuvent être facilement corrêlées avec celles des groupes Colomi et Itacolomi déjà mentionnés.

L'absence presque complète d'affleurements dans les plaines et les dépressions, la rareté des voies de pénétration et l'épais manteau d'intempérisme rendent difficile la réalisation d'une carte géologique détaillée.

Les affleurements, peu fréquents, qui se localisent presque toujours sur les lignes de crête, dominant les dépressions de 80 à $120 \mathrm{~m}$ (Morro do Teiú), sont essentiellement 
représentés par du minerai de fer et de titane, dur, compact et très résistant, et quelques gneiss quartzeux. Leur direction générale varie de NS à N $35^{\circ} \mathrm{E}$, comme au lieu-dit Ponta da Serra où ont été observées des couches subverticales.

Les gneiss quartzeux encaissants le minerai sont clairs, d'un grain moyen à grossier et d'une structure bien mise en evidence par des alternances de lits de quartz et de feldspath et d'autres où de la biotite, toujours en faible quantité, s'associe aux minéraux précédents. Des enrichissements locaux en microcline, sous la forme d'agrégats lenticulaires donnent à la roche un aspect d'embrêchite.

La résistance des gneiss à l'érosion, bien moindre que celle du minerai, explique la rareté de leurs affleurements, la roche formant le piedmont des collines où se localisent les minéralisations et les dépressions entourant celles-ci.

Au point de vue tectonique, la constance des pendages vers l'WNW, en opposition à ceux de direction Est, qui peuvent être observés dans la zone du Báxão do Calixto et au lieu-dit Pedra Comprida (hors de la carte), conduit à penser que la minéralisation se localise sur le flanc oriental d'un synclinal serré, dont l'axe passerait à l'Ouest de la limite de la carte.

Régionalement, cette conclusion s'encadre parfaitement avec les observations de A. L. Caldasso et al. (1973) qui ont cartographié, à l'Ouest de la Serra da Boa Esperança, un synclinal, dont l'axe prolongé coïnciderait avec celui de la structure précédemment mentionnée.

2. AFFLEUREMENTS MINERALISES - GENERALITES Trois couches de minerai ont été identifiées, ainsi que diverses lentilles plus petites. Ce sont d'Ouest en Est, les deux dernières étant parallèles, celles de:

-Morro Vermelho

- Morro da Carlota - Morro da Pedra Comprida Ouest

- Morro do Teiú - Morro da Pedra Comprida Est - Morro Velho.

Les pentes des élévations sont recouvertes par deș blocs et fragments éboulés reposant sur un sol argileux brun rouge.

Les couches de minerai, à l'exception de celle du Morro Vermelho, présentent une grande continuité longitudinale. Elles peuvent ainsi être suivies sur plus de $5 \mathrm{~km}$, formant sans aucun doute un chapelet irrégulier.

Leur épaisseur moyenne, du fait que les contacts du minerai avec la roche encaissante sont masqués par les éboulis, ne peut être déterminée exactement. Gependant, de nombreux affleurements (Morro do Teiú, da Pedra Comprida - Fig. 2 - et Velho), larges d'une cinquantaine de mètres, permettent de déduire qu'en fonction du pendage général, l'épaisseur probable de la minéralisation doit être de l'ordre d'une trentaine de mètres tout au moins dans la partie centrale des lentilles.

Le minerai, compact, a une texture grenue. Il est formé de grains supérieurs à $1 \mathrm{~mm}$ et de couleur noirâtre, brunâtre ou rougeâtre.

En certains points la magnétite prédomine, comme au Morro do Teiú, en d'autres, comme sur le versant Sud de la Pedra Comprida, l'ilménite parvient à la remplacer presque totalement.

3. MORRO DO TEIÚ Comme on vient de le voir, la magnétite y prédomine dans le minerai. Celui-ci est décrit par A. L. Caldasso et al. (1973) comme de "densité élevée, éclat métallique, couleur noire à rouge si oxydé et fort magnétisme". 


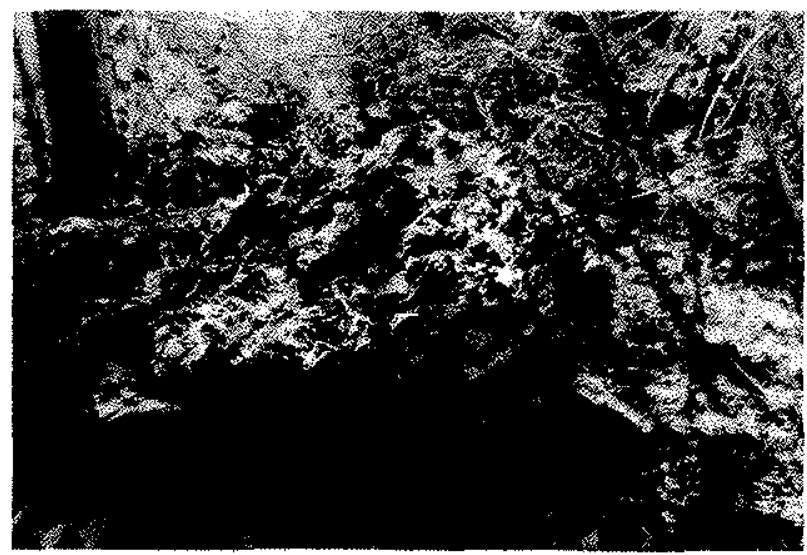

Figure 2 - Affleurement de minerai titanifère dans la caatinga sur les pentes du Morro da Pedra Comprida

Un échantillon provenant de ce Morro, calibré par criblage entre 35 et 80 meshes et soumis à une séparation magnétique et electromagnétique (inclinaison latérale et frontale de $15^{\circ}$ ), a donné:

$65 \%$ de produit magnétique (magnétite + mixtes)

$30 \%$ attirable à $0,1-0,2 \mathrm{~A}$ (ilménite + mixtes)

$2 \%$ at tirable entre 0,3 et $1,6 \mathrm{~A}$ (minerais opaques associés à la gangue)

$3 \%$ non at tirable (gangue).

Magnétite et ilménite subordonnée sont ainsi les constituants essentiels de ce minerai.

Au point de vue microscopique, le minerai est très homogène et contient souvent plus de $90 \%$ de minéraux opaques, la gangue étant de chlorite, calcédoine et hydroxydes de fer sans quartz ou minéraux ferromagnésiens. Les produits secondaires sont peu importants ét représentés par la limonite et du leucoxène.

La magnétite, en grains irrégulièrement distribués, mais sans orientation marquée, est le minéral le plus abondant. Généralement pure, elle présente quelquefois des lamelles d'exsolution d'ilménite, ou un début de martitisation.

L'ilménite est identique à celle de la Pedra Comprida qui sera décrite postérieurement. L'hématite est le minéral le moins abondant, soit primaire, soit provenant de l'altération de la magnétite.

4. MORRO DA PEDRA COMPRIDA Au voisinage de la route venant de Campo Alegre la minéralisation se manifeste par de grands blocs épars sur le sol et dépassant plusieurs mètres cubes au Nord. Ils sont recouverts par une croûte brun rougeâtre à noirâtre, ou rougeâtre et limonitique.

Affleurement Ouest. Le minerai y est composé de grains irréguliers parfois allongés en lamelles ou noyés dans un ciment de limonite superficielle. En d'autres points ils sont en mosaïque et presque jointifs. La gangue est peu abondante, surtout micacée, ou de feldspaths ferruginisés.

Parfois les blocs présentent une patine vernissée extérieure, le plus souvent marron à brune, formant un cortex irrégulier très chagriné. Localement des hydroxydes de fer y soulignent la linéation.

Affleurement Est. Son minerai rappelle le précédent, mais quelquefois des cavités spongieuses y sont remplies de limonite ou d'hématite pulvérulente. Des cristaux de gangue blanche sont noyés dans la masse. Il existe tous les termes de transition d'une roche ferrifêre au minerai massif, certains présentant une schistosité très nette. 
L'absence presque complète de magnétisme, remarquée sur le terrain pour les échantillons du Morro da Pedra Comprida a conduit à penser qu'ils différaient profondément de ceux du Morro do Teiú précédemment décrits. Leur étude détaillée constituera l'objet du chapitre suivant, explicitant les caractéristiques de ce minerai titanifère assez exceptionnel au Brésil.

Une séparation magnétique et electromagnétique sur un échantillon pratiquement massif, calibré entre 0,1 et $0,5 \mathrm{~mm}$, dans les mêmes conditions d'utilisation qu'au paragraphe précédent, a donné:

$3,7 \%$ de produit magnétique

$20,6 \%$ attirable à $0,1 \mathrm{~A}$

$64,1 \%$ attirable à $0,3 \mathrm{~A}$

$11,2 \%$ attirable à $0,5 \mathrm{~A}$

$0,2 \%$ attirable à $0,8 \mathrm{~A}$

$0,1 \%$ attirable à 1 et $1,5 \mathrm{~A}$

$0,1 \%$ non magnétique.

A l'inverse du Morro do Teiú, ces résultats démontrent la prédominance de l'ilménite et des ses mixtes sur la magnétite, la gangue, très peu abondante, ayant été en partie éliminée lors de la préparation de l'échantillon.

Etude du minerai du Morro da Pedra Comprida 1. TEXTURE GENERALE Malgrè de nombreuses variations de détail, la texture du minerai de Pedra Comprida peut être ainsi synthétisée: de grands cristaux d'ilménite magnésienne, plus ou moins corrodés, sont entourés par un ciment très homogène constitué généralement d'hématite à exsolution d'ilménite, le tout étant plus ou moins disloqué et envahi par de la gangue. La magnétite est rare (Fig. 3).

Figure 3 - Aspect général du minerai. Grands cristaux d'ilménite dans le ciment. Lumière naturelle (le trait correspond à $0,1 \mathrm{~mm}$ )

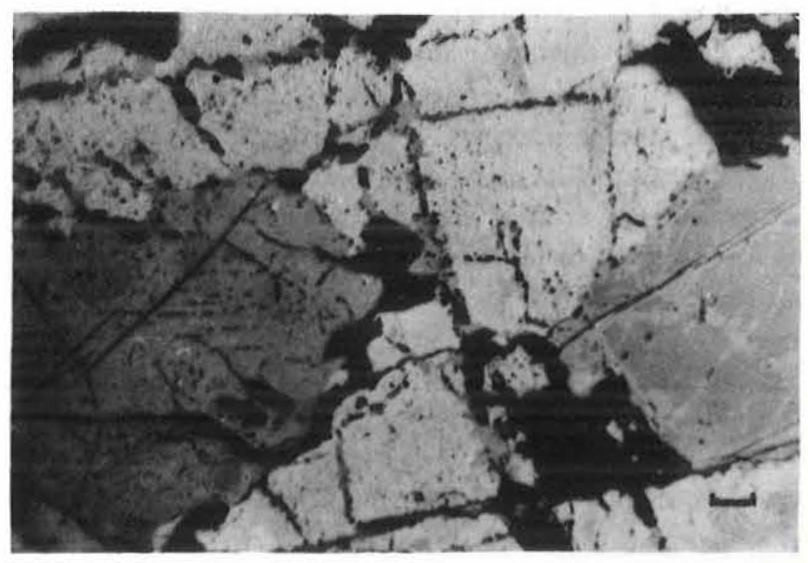

Nous examinerons ci-après les constituants de ce minerai dont la paragenèse est très simple. Leur étude a été réalisée à partir de l'observation d'une quinzaine de sections polies normales.

\section{ILMENITE EN GRANDS CRISTAUX}

2.1. Description microscopique Ces grands cristaux, dont la taille varie de 0,5 à $2,5 \mathrm{~mm}$ sont généralement arrondis, très rarement à peine anguleux (Fig. 4). Leur contact avec le ciment est quelquefois linéaire, brusque, mais le plus souvent irrégulier et graduel. 


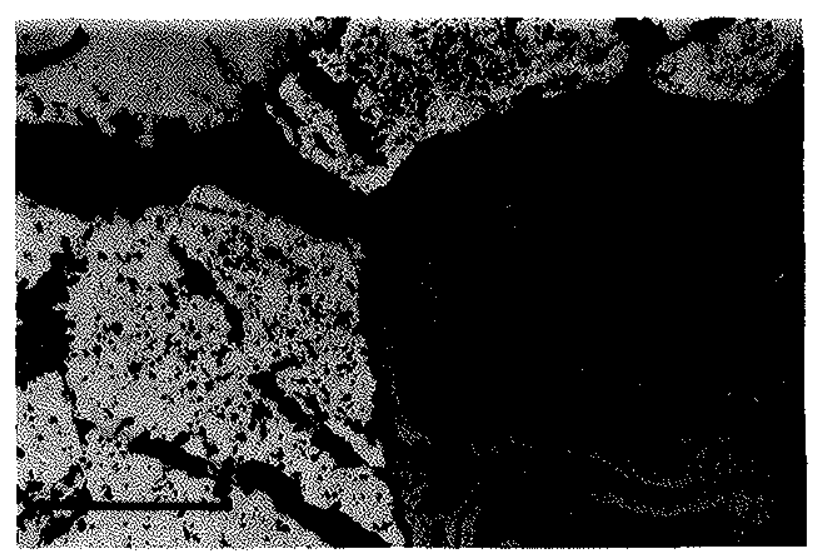

Figure 4-Grand cristal d'ilménite magnésienne. Des plages plus claires, ferrifêres, sont visibles le long des fissures. Nicols croisés à $50^{\circ}$ (le trait correspond à $0,1 \mathrm{~mm}$ )

Ils polarisent en gris bleu très foncé et présentent dans leurs fissures et sur leurs bords de nombreuses réflexions internes rouge brun à rouge sang laissant supposer qu'il s'agit d'un terme magnésien de la série ilménite-giekielite (B. Cervelle, 1967). Cette observation est confirmée par la relative rareté des aiguilles d'exsolution d'hématite, indiquant l'absence d'un excès de fer pendant leur formation.

La présence d'ilménite magnésienne n'étant pas commune dans les gites en roche, il a été jugé intéressant d'étudier en détail celle des grands cristaux afin de mieux préciser leurs caractères, tant au point de vue métallographique que diffractométrique et chimique.

2.1.1. Pouvoir réflecteur et microdureté Une série de mesures de pouvoir réflecteur en lumière blanche, en utilisant un étalon de pyrite a permis d'obtenir une valeur moyenne de $18 \pm 1 \%$.

Comme terme de comparaison, le pouvoir réflecteur de grands cristaux d'hématite existant en d'autres sections polies, et qui seront décrits plus loin, a été trouvé égal à $28,5 \%$, en bon accord avec les données de la littérature spécialisée.

La microdureté Knoop, pour une charge de $100 \mathrm{~g}$ est de $1881 \mathrm{~kg} / \mathrm{mm}^{2}$, en opposition avec celle du ciment encaissant: $1098 \mathrm{~kg} / \mathrm{mm}^{2}$. Celui ci sera décrit ultérieurement. Rappelons que, dans les mêmes conditions, la blende de Picos de Europa (Santander, Espagne) a une microdureté de $183,7 \mathrm{~kg} / \mathrm{mm}^{2}$.

2.1.2. Constitution des cristaux De nombreux cristaux présentent de larges plages brun vert à brunâtre qui polarisent du gris bleu clair au marron ou pourpre foncé et qui sont limitées par des fissures et des bandes à peine plus claires gris bleuâtre en lumière naturelle; on y note de nombreuses réflexions internes en lumière polarisée. Cet assemblage correspond sans doute à un changement de composition chimique durant le dépôt: les plages centrales sont plus riches en magnésium que les bordières où la proportion de fer augmente légérement.

Les aiguilles d'hématite d'exsolution sont rares et parallèles aux bandes claires (Fig. 5). Il en est de même des lamelles de macle qui se repèrent généralement au voisinage des zones altérées.

Certains grains présentent de curieuses vermiculations (Fig. 6). Il s'agit sans doute d'une intercroissance particulière entre minéraux de la même famille (Fig. 7).

2.1.3. Inclusions Les grands cristaux renferment sporadiquement, très localement, de petites inclusions rectangulaires aux angles arrondis et de très fines aiguilles parallèles 
Figure 5-Aiguilles d'exsolution d'hématite dans l'ilménite magnésienne. Nicols croisés à $45^{\circ}$ (le trait correspond à $0,1 \mathrm{~mm}$ )
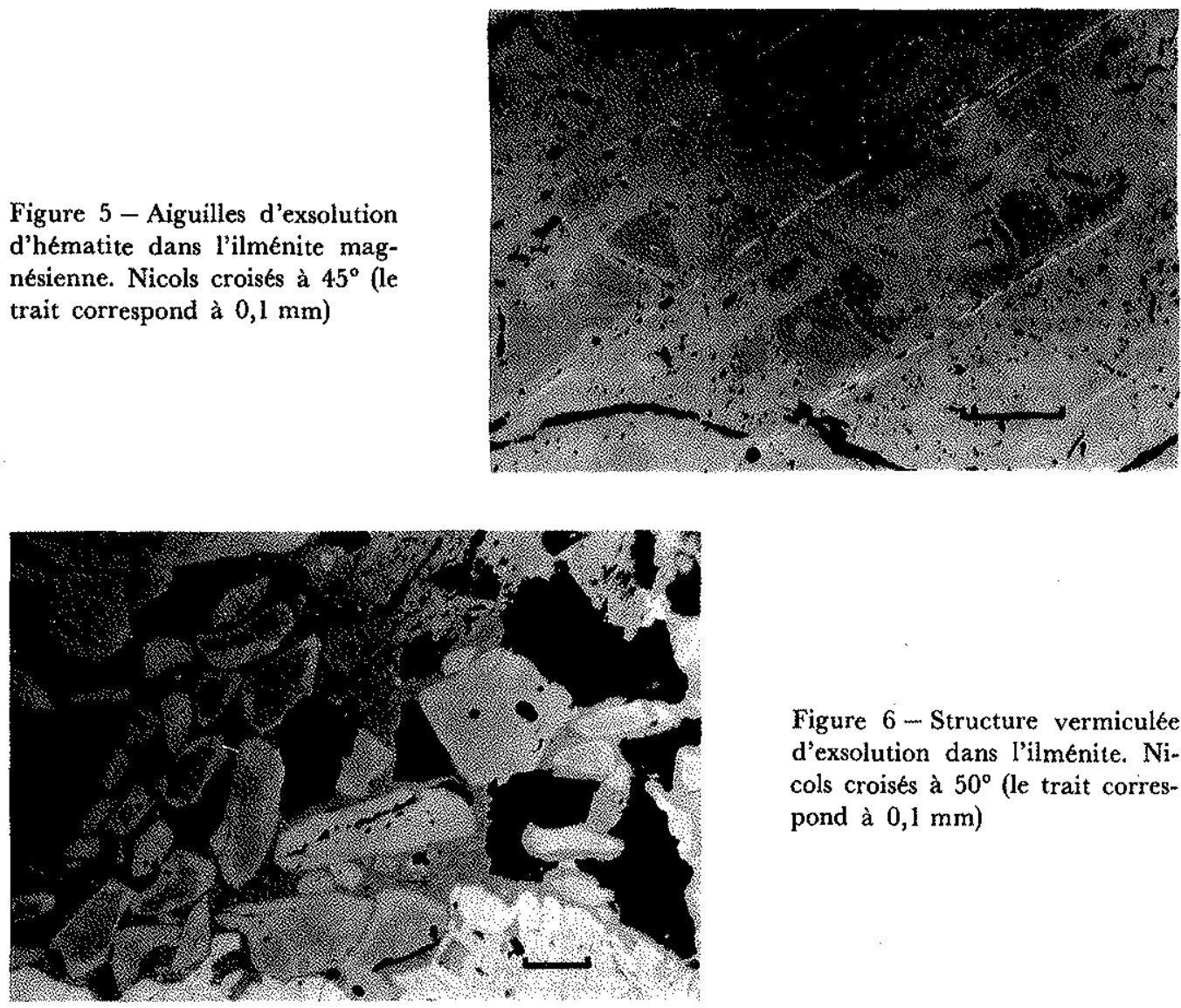

Figure $6-$ Structure vermiculee d'exsolution dans l'ilménite. Nicols croisés à $50^{\circ}$ (le trait correspond à $0,1 \mathrm{~mm}$ )

Figure 7-Structures de croissance dans l'ilménite magnésienné (le trait correspond à $0,1 \mathrm{~mm}$ )

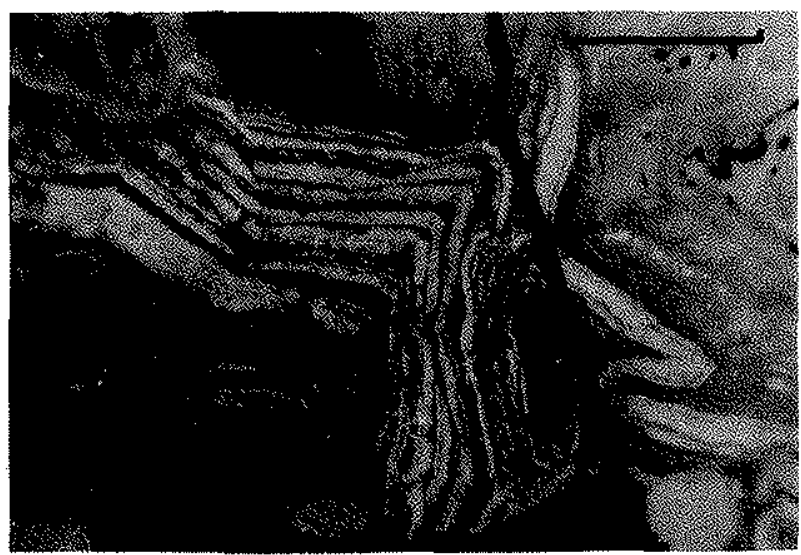

d'hématite se terminant en ogive. Il y a quelques coalescences et des élargissements faisant passer en zig-zag de l'une à l'autre et donnant des zones graphiques vermiculaires. Ces aiguilles se montrent quelquefois à fort grossissement renfermant de petits ilots d'ilménite. Etant d'orientation differente dans de grands cristaux jointifs, elles prouvent que le minerai initial avait une structure grenue. 
2.1.4. Fissures Les grands cristaux sont fréquemment parcourus par d'infimes fissures parallèles ou par des cassures irrégulières plus importantes. Ils sont envahis par la gangue, ce qui est le cas le plus général, et par le ciment qui y dessine de petites iles irrégulières. Les fissures se prolongent dans ce dernier, ou réciproquement (affleurement Est), alors qu'en d'autres points elles s'arrêtent au contact des grands cristaux. Leur bord est généralement denticulé.

2.2. Etude diffractométrique L'étude diffractométrique des grands cristaux d'ilménite a été réalisé afin de déterminer le déplacement des raies que devait produire la présence supposée du magnésium, par rapport à de l'ilménite pure.

On trouvera dans le Tableau I les données des fiches ASTM relatives à l'hématite, la geikielite et l'ilménite, celles de B. Cervelle (1967) pour l'ilménite et la geikielite et enfin celles du minéral de Campo Alegre. Notons que l'échantillon relatif á ce dernier inclut un peu d'hématite et d'ilménite commune qui n'ont pu être éliminées durant la prépa* ration.

La correspondance entre les valeurs $Q=\frac{1}{d_{h \mathrm{k} 1}^{2}}$ observées et calculées de cet échantillon est donnée dans le Tableau II.

Le calcul des paramètres de la maille élémentaire a donné:

$$
\begin{aligned}
& a_{0}=5,056 \AA \pm 0,003 \\
& b_{0}=13,801 \AA \pm 0,003
\end{aligned}
$$

TABLEAU I

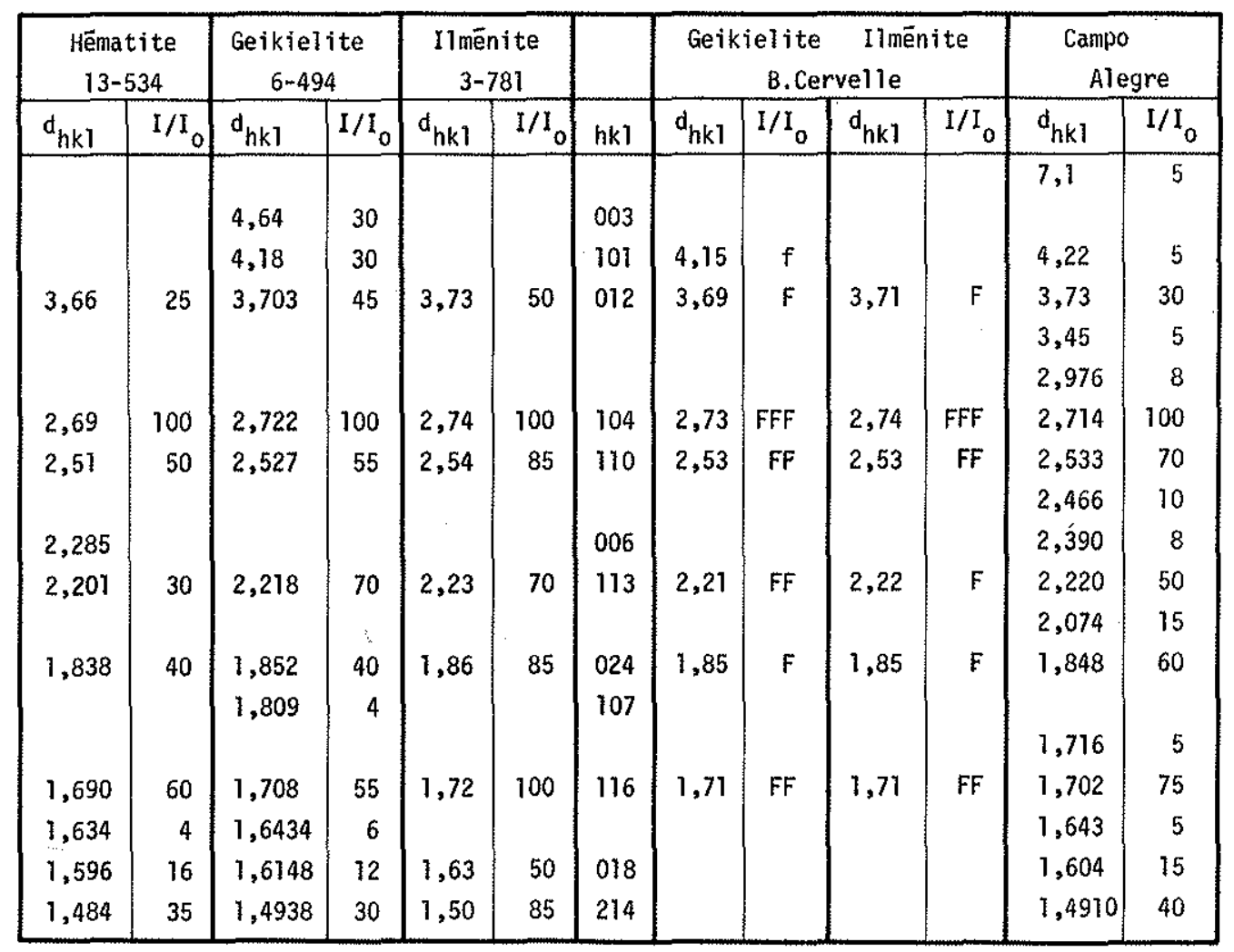


TABLEAU II

\begin{tabular}{|l|r|r|r|l|}
\hline$d_{h k l}$ & $I_{0} / I_{\text {hkl }}$ obs. & hkl & $Q_{\text {hkl }}$ calc. \\
\hline 4,22 & 5 & 0,0561 & 101 & 0,0573 \\
3,73 & 30 & 0,0719 & 012 & 0,0731 \\
2,714 & 100 & 0,1368 & 104 & 0,1365 \\
2,533 & 70 & 0,1559 & 110 & 0,1563 \\
2,220 & 50 & 0,2029 & 113 & 0,2037 \\
1,848 & 60 & 0,2928 & 024 & 0,2924 \\
1,702 & 75 & 0,3452 & 116 & 0,3453 \\
1,643 & 5 & 0,3704 & 211 & 0,3699 \\
1,604 & 15 & 0,3887 & 018 & 0,3881 \\
1,492 & 40 & 0,4492 & 214 & 0,4487 \\
\hline \multicolumn{5}{|c}{$\mathrm{Z}=2$} \\
\hline
\end{tabular}

La maille de la geikielite est: $a_{0}=5,054$ et $c_{0}=13,898$; celle de l'ilménite est $a_{0}=5,079$ et $c_{0}=14,135$ et celle de l'hématite: $a_{0}=5,032$ et $c_{0}=13,737$ selon l'ASTM, en $\AA$. Il est donc possible d'écrire:

$$
\begin{array}{r}
a_{0} \text { hématite }<a_{0} \text { geikielite } \leqslant \text { minéral de Campo Alegre }<a_{0} \text { ilménite } \\
\qquad c_{0} \text { geikielite }<\text { minéral de Campo Alegre }<c_{0} \text { ilménite }
\end{array}
$$

Le minéral de Campo Alegre s'encadre donc parfaitement entre les deux termes extrêmes de la série ilménite-geikielite et sa nature d'ilménite magnésienne est pleinement confirmée par les résultats diffractométriques.

2.3. Dosage du magnésium Une analyse spectrographique qualitative de l'échantillon soumis à l'étude diffractométrique a mise en évidence: Ti-Fe, Si-Al-Mg-Cu-Mn-Co-Ni.

La quantité de magnésium présente dans les grands cristaux n'a pu être déterminée exactement, les fragments prélevés sur les sections polies sous la loupe binoculaire renfermant encore des fragments de ciment et donc apportant du fer en excès. Le dosage de ce mélange a donné $4 \% \mathrm{MgO}$ et correspond ainsi à une teneur par défaut.

En conclusion: les grands cristaux doivent ainsi être classés comme appartenant à une ilménite magnésienne, leur teneur en $\mathrm{MgO}$ n'est pas suffisante pour les faire ranger dans le groupe de la geikielite.

3. CIMENT Ainsi qu'il a été vu précédemment, les grands cristaux sont noyés dans un ciment gris plus clair, d'un ton plus jaune que l'ilménite et toujours plus poreux que celle-ci au polissage.

A faible grossissement, il présente une structure réticulée, mouchetée en lumière polarisée: c'est une mosaïque très fine sans orientation particulière. A fort grossissement il montre une structure d'exsolution plus ou moins fine à faciès variable, très caractéristique, d'ilménite ordinaire dans l'hématite. Celle-ci, totalement indépendante des grands cristaux se mêle localement à des ponctuations isolées et des veinules d'ilménite, le tout étant largement envahi par la gangue. 


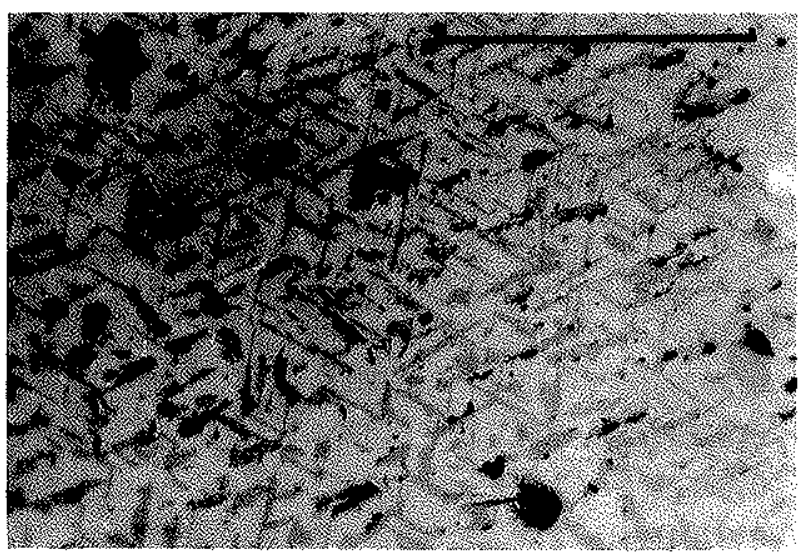

Figure 8-Exsolution fine d'ilménite dans l'hematite du ciment. Lumière naturelle (le trait correspond à $0,1 \mathrm{~mm}$ )

A l'intérieur du ciment il est possible de noter les faciès suivants:

-Le plus répandu correspond à une exsolution rappelant une texture graphique (Fig. 8). Elle est constituée par de petits bâtonnets parallèles, souvent discontinus, par files de deux, des ponctuations et des taches irrégulières aux contours dentelés se recoupant suivant des angles variables, selon l'inclinaison de la section par rapport au réseau (Fig. 9).

-Occasionnellement un large développement de l'ilménite relègue, au contraire du faciès précédent, l'hématite au second plan. Cette structure est peu courante.

- Localement de grandes lamelles d'ilménite parallèles multimillimétriques donnent un aspect compartimenté au ciment.

-En d'autres points les lamelles précédentes se recoupent suivant deux réseaux, englobant entre elles des ilots d'exsolution très fine habituelle.

-Enfin de nombreux grains polyédriques plus ou moins arrondis d'ilménite sont épars dans le ciment. Le plus généralement ils sont isolés, quelquefois groupés et voisinent avec la structure d'exsolution la plus commune qu'ils ne substituent que rarement. Les plages à grains orientés sont rares. Il s'agit toujours de syncristallisation avec l'exsolution.

Des chapelets de grains allongés d'ilménite partent d'un grand cristal et le relient à un autre ou s'effacent dans le ciment.

Enfin, divers grains poreux d'ilménite présentent des réflexions internes et quelquefois un début d'alteration rappelant celui des grands cristaux (Fig. 10). Il s'agit peut être de reliques en voie de digestion par le ciment ou de remaniement de celui-ci.

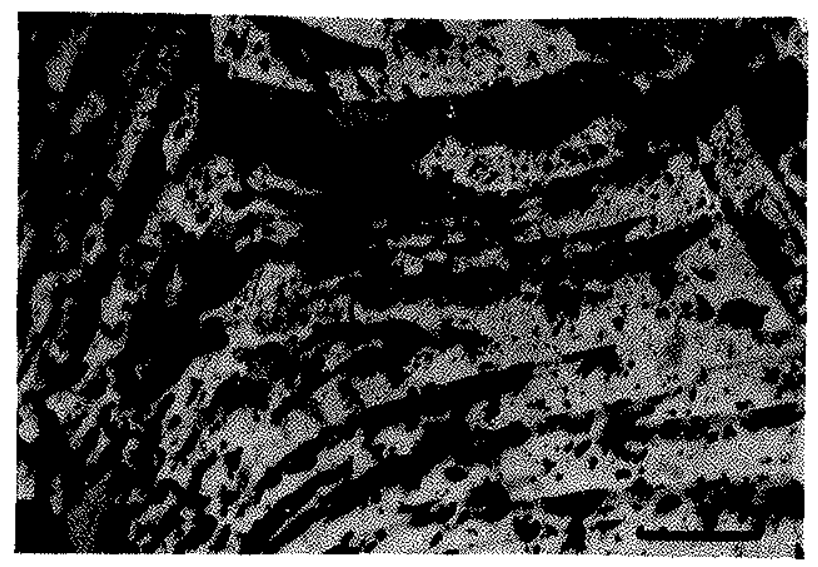

Figure 9-Lamelles tordues d'ilménite dans l'hématite. Lumière naturelle (le trait correspond à $0,1 \mathrm{~mm}$ ) 
Figure 10-Ilménite en voie de decomposition dans le ciment. $\mathrm{Ni}$ cols croisés à $80^{\circ}$ (le trait correspond à $0,1 \mathrm{~mm}$ )

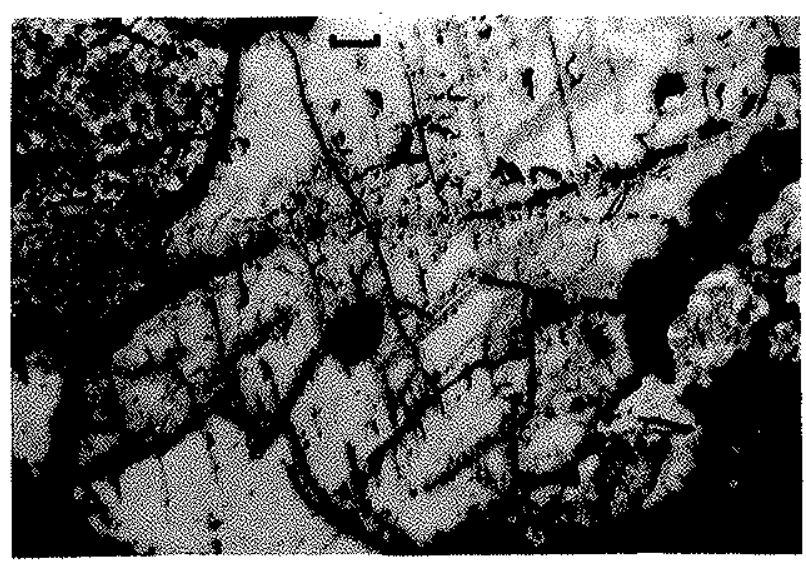

La fracturation du ciment s'est souvent faite le long des veinules d'ilménite dont les grains plus ou moins allongés flottent dans la gangue entre les bords du ciment.

4. AUTRES MINERAUX 4.I. Hématite Ce minéral a déjà été partiellement étudié lors de la description du ciment. Il est aussi très fréquent dans la gangue sous forme de petites paillettes disséminées, en trainées ou en réseau irrégulier, mais sans rosettes, donnant une teinte rouge sang à l'ensemble en lumière polarisée.

On trouve également beaucoup d'hématite en paillettes et lamelles très fines au bord des fragments du ciment, à leur limite avec la gangue, formant des effilochures comme si les morceaux avaient été arrachés suivant plusieurs directions et non cassés.

Enfin, assez exceptionnellement, l'hématite forme de grands cristaux dans quelques échantillons (Affleurement Est, Fig. 11). Elle est plus claire que l'ilménite, avec de fines macles parallèles se recoupant suivant deux réseaux. Elle est à l'origine de la goethite qui sera décrite ultérieurement.

4.2. Magnétite Peu abondante, elle se présente généralement en petites îles arrondies ou polyédriques curvilignes, éparses irrégulièrement dans le ciment.

Un début de martitisation peut être observé localement.

Figure 11 - Hématite en grands cristaux. Lumière naturelle (le trait correspond à $0,1 \mathrm{~mm}$ )

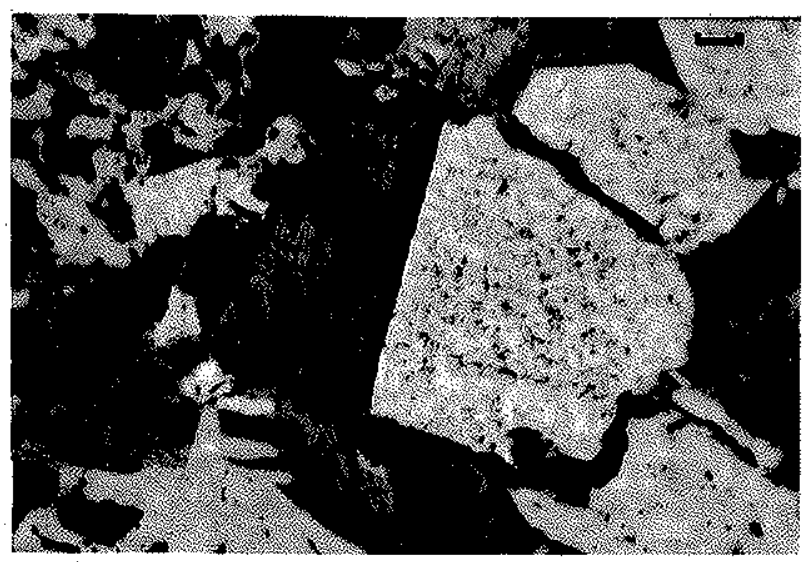




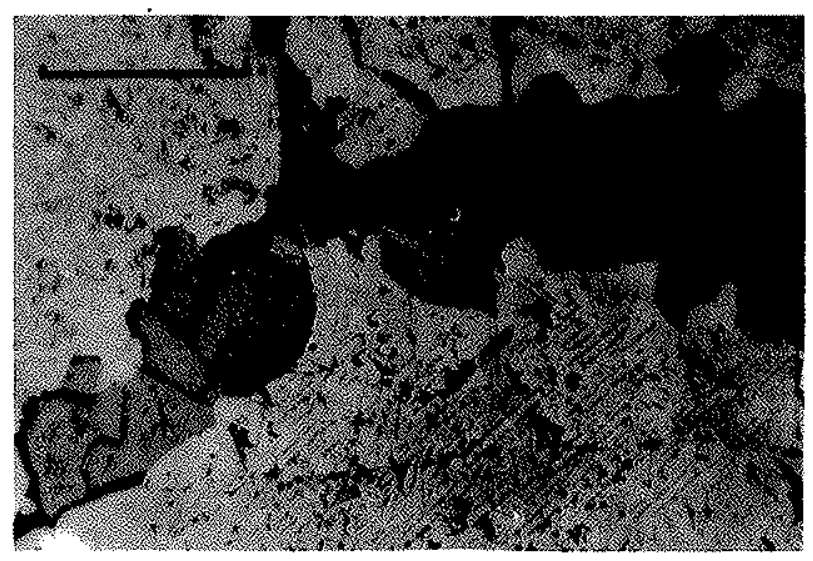

Figure 12 - Filonnet de rutile dans le ciment. Lumière naturelle (le trait correspond à $0,1 \mathrm{~mm}$ )

4.3. Rutile Très rare, en relief, possédant des reflets jaune orangé intense, il forme des veinules de petits cristaux irréguliers traversant le ciment (Fig. 12).

4.4. Leucoxêne Beaucoup plus abondant que le rutile, il constitue de minces croûtes réniformes autour de fragments du ciment et de certains grains d'ilménite dans les fractures desquels il pénètre.

Légèrement plus gris que l'ilménite, beaucoup plus poreux et plus tendre au polissage, il présente d'intenses réflexions rouges en lumière polarisée.

\subsection{Sulfures Aucun sulfure n'a été observé ni dans la gangue ni dans le minerai.}

4.6. Goethite Plus sombre que l'hématite en lumière naturelle, des réflexions internes jaune ocre en masquent l'anisotropie en lumière polarisée (Affleurement Est).

La goethite se présente en fibres parallèles, radiées ou en queue de cheval, plus rarement en amas compacts irréguliers généralement entourés de grains de minerai non décomposé. Elle dérive de l'altération de certains d'entre eux sans loi définie. On trouve également de petites aiguilles de goethite éparses dans le mica de la guangue, une partie de celles-ci disposées en réseau paraissant être le squelette résiduel d'un minéral disparu.

4.7. Gangue La gangue, souvent colorée en rouge ou brun par de l'hématite, de la goethite ou par les réflexions dûes aux oxydes voisins, est tendre, essentiellement formée de mica et de feldspath sans quartz.

Le mica, en peignes, rosettes et taches irrégulières, est toujours en paillettes ṕlus ou moins colorées par de fines inclusions de minéraux opaques ou par les réflexions des fragments voisins identiques.

Les feldspaths sont kaoblinisés, tendres (dureté inférieure à 5), blanc laiteux, en masses compactes plus ou moins fortement teintées par d'infimes ponctuations d'oxydes de fer et montrent parfois quelques faces cristallines.

5. STRUCTURE DU MINERAI L'ensemble ilménite en grands cristaux-ciment est plus ou moins fendillé et plus ou moins envahi par la gangue. Diverses structures résultent de l'intensité avec laquelle ces deux phénomènes ont joué.

Il est difficile de parler de cataclase. Le minerai parait plutôt avoir été comprimé puis s'être ensuite légerement disloqué, ses morceaux restants très souvent proches les uns 
des autres. C'est le plus ou moins grand développement de la gangue qui donne ses caractéristiques à la structure.

Seulement quelques lamelles ployées d'ilménite attestent des efforts tectoniques postérieurs à leur cristallisation (voir Fig. 9).

On peut schématiquement observer les structures suivantes:

Structure brêchique. Les fragments de minerai y ont des contours assez linéaires et sont séparés par de minces filets de gangue. La trituration est antérieure à cette dernière qui entraine dans les fissures des lambeaux étirés d'hématite.

Structure pseudorubannée. Elle est dûe à une trituration formant des fragments lamellaires à partir de fissures parallèles. Localement le minerai présente des fentes suivant deux réseaux à $120^{\circ}$ et une porosité élevée au polissage.

Structure cariée ou effilochée. Lorsque la gangue devient importante, ou prédomine, les débris de minerai restants, qui peuvent y être isolés, prennent une allure cariée, spongieuse ou effilochée. Les grands cristaux d'ilménite sont également plus ou moins dissociés et quelquefois réduits à des échardes. Les fissures de la première structure sont alors remplacées par des passées larges, plus ou moins régulières, de gangue.

Le passage de l'une à l'autre de ces structures est rapide et peut être observé dans une même section par diminution de la proportion de gangue par exemple.

En résumé, l'histoire du minerai parait être la suivante:

-Dépôt des grands cristaux d'ilménite, magnésiens pendant l'essentiel de leur croissance, un peu plus ferrifères avec début de résorbtion, à la fin de celle-ci.

- Dépôt du ciment, c'est à dire d'une exsolution d'ilménite normale très fine dans l'hématite, avec magnétite rare. Très localement de l'hématite s'individualise en grands cristaux. Une légère fissuration locale fait pénétrer le ciment dans les fentes de certains grands cristaux.

-Fracturation de l'ensemble, et envahissement par la gangue.

-Altération météorique, très peu accentuée, en surface.

Origine du gite Les études géologiques réalisées à l'échelle régionale ont conduit leurs auteurs, précédemment cités, à conclure que la série lithologique affleurant dans la région de Campo Alegre de Lourdes est identique, dans ses grands traits, à celle de la Serra do Espinhaço, où existent des itabirites.

Gependant A. L. Caldasso et al. (1973) signalent qu'au Morro do Teiú “quelques echantillons de minerai présentent une texture résiduelle rappelant l'altération des minéraux silicatés ferromagnésiens, avec des intercalations de lits de magnétite, qui suggèrent une possible origine à partir de corps basiques intrusifs". Ces derniers auraient fait intrusion dans des sédiments qui ont été ensuite métamorphisés et constituent l'actuel Précambrien B.

Donc, si d'une part, le minerai de Pedra Comprida a été comparé aux itabirites du géosynclinal de l'Espinhaço, d'autre part quelques unes de ses caractéristiques le rapprocheraient des dépôts orthomagmatiques.

Avant d'essayer d'élucider la genèse de ce gîte, et d'infirmer ou confirmer les hypothèses précédentes, nous rendrons compte du résultat des compilations récentes (J. Lombard, 1957; P. Routhier, 1963). Celles-ci, traitant des grands gîtes de titane mondiaux, s'accordent pour mettre en évidence:

-leur association avec les complexes alcalins, les carbonatites ou les grands ensembles basiques: gabbro, norite et anorthosites souvent encaissés dans des séries charnockitiques;

-leur localisation dans les boucliers précambriens; 
-leur allure plus ou moins stratiforme avec minerai assez souvent rubanné, présentant toujours des structures d'exsolution.

En outre, les auteurs précédents signalent:

-que le rutile est la forme d'oxyde la plus stable en milieu acide (exception faite des carbonatites, telle Magnet Cove, Arkansas). Dans les gîtes de faible importance liés au granite, l'ilménite s'associe aux terres rares (lanthanides), zirconium, niobium et tantale;

-que plus la cristallisation est tardive, plus le minerai est riche en ilménite par rapport à la magnétite (comme dans les gîtes de Tellnes et Storgangen du district d'Egersund en Norvège du Sud). Pour E. R. Rose ces gisements sont tardi-magmatiques (1969);

-qu'enfin le métamorphisme de concentrations titanifêres en roches basiques produit du rutile qui peut migrer dans les strates voisines, laissant un abondant reliquat d'hématite dans l'ilménite.

Le minerai, de granulation homogêne, est généralement constitué par de l'ilménite prédominante du type ferri-ilménite (R. Chevallier et al., 1955), avec lamelles d'hématite (Lacs Allard et Tio à Québec) et magnétite en quantité variable ( $22 \%$ au Lac Allard). Quelques sulfures y sont associés. Localement existe un peu de vanadium dans le minerai, mais il ne constitue pas une caractéristique générale de celui-ci.

L'absence de phosphore différencie les gîtes liés aux anorthosites de ceux liés aux carbonatites.

Les minéralisations incluses dans les pyroxénites ne semblent pas à ce jour constituer des concentrations économiquement exploitables (J. Boulanger, 1959).

Enfin, les gites sont des sills ou des lopolithes sans relation avec le métamorphisme du cadre encaissant, bien que P. Hammond (1952) parle de "mise en place sous forme de sills et épais feuillets introduits dans les anorthosites". De même E. Rose (1969) signale l'existence d'extrusion en raison de l'observation de phases figées de roches anorthosiques.

En fonction des données bibliographiques précédentes on peut déduire:

-que l'absence de lits quartzitiques et la présence généralisée de textures d'exsolution observées dans toutes les sections polies, ainsi que les variations de composition relativement rapides dans une même couche (proportion ilménite/magnétite entre autres) doivent faire écarter toute assimilation avec des formations itabiritiques.

eque la pauvreté en phosphore et la présence de vanadium (détecté par des analyses de la CPRM) dans le minerai de Campo Alegre de Lourdes éliminent une possible filiation à partir de carbonatites.

-que l'absence de terres rares et de rutile, ainsi que de niobium et de tantale tliminent de même toute relation génétique avec des roches acides.

-qu'enfin une série de caractéristiques du gîte de Pedra Comprida plaident pour une origine magmatique. Entre autres:

-la forme en lentille très aplatie du gîte encaissé dans des formations précambriennes rappelle celle de nombreux grands gisements précédemment signalés,

-l'absence de quartz dans la gangue, essentiellement formée de mica et de feldspaths kaolinisés, incite à penser à une matrice basique fortement transformée,

-l'omniprésence des textures d'exsolution déjà signalées,

- les variations notables de composition entre les Morros do Teiú et de Pedra Comprida, qui peuvent être mises sur le compte d'une différenciation dans le temps et/ou l'espace.

Dans la classification de P. Routhier (1963) le type 13 "gîtes d'hématite à ilménite en exsolution" comme ceux des Lacs Allard et Tio, serait le plus proche de celui du gisement de Campo Alegre. 
Cependant à l'encontre de tous les critères précédents, il convient de remarquer:

-qu'il n'existe au voisinage de Campo Alegre aucune série anorthosique ou charnockitique analogue à celle dans laquelle sont encaissés tous les grands gisements précédemment mentionnés.

-qu'il n'y a pas de sulfures dans le minerai.

-qu'aucune ilménite magnésienne n'est signalée dans les gisements du type Lac Tio ou Allard où le constituant essentiel est la ferri-ilménite (R. Chevallier et al., 1955). Par contre de l'ilménite magnésienne est fréquente dans les roches basiques, comme les gabbros ou les kimberlites par exemple (M. G. Bardet, 1973).

En fonction des observations précédentes il semble donc que l'on puisse considérer actuellement le gîte de Campo Alegre comme d'origine magmatique, mais de type exceptionnel.

Quant à sa genèse, elle reste présentement assez incertaine. Les travaux de recherche minière devant être réalisés sur les affleurements permettront sans doute de mieux connaître la roche encaissant le minerai et les contacts de celle-ci avec le gneiss stérile.

Il est possible qu'une masse de minerai titanifère différenciée en profondeur à partir d'un complexe basique ou ultrabasique se soit injectée dans une zone de faiblesse du socle. Si la présence d'ilménite magnésienne et le voisinage, relatif, du massif ultrabasique de São José do Piauí permettent de sustenter partiellements l'hypothèse précédente, on manque encore de données de terrain pour la confirmer.

Quant à l'âge de la mise en place du gîte, il reste à définir si l'intrusion a été pré ou post-métamorphisme. La structure générale du gisement se ployant aux ondulations tectoniques régionales soutient la première hypothèse, alors que l'absence de rutile et la présence de grands cristaux d'ilménite presque pure témoignent en faveur de la seconde.

Les travaux miniers, actuellement en cours, permettront peut être de lever ces indéterminations.

\section{BIBLIOGRAPHIE}

BARBOSA, O. - 1965 - Geologia das folhas Remanso-Sento Sé, Bahia. Projeto Médio São Francisco DNPM/Prospec. Relatório inédito

BARDET, M. G. - 1973 - Géologie du diamant. Première partie: généralités. Mém. B.R.G.M. 83: $235 \mathrm{pp}$.

BOULANGER, J. - 1959 - Les anorthosites de Madagascar. Ann. géol. de Madagascar. Fasc. XXVI: $71 \mathrm{pp}$.

BRUNI, M. A. L., ALMEIDA, J. T. de et BRUNI, E. G. - 1974 - Carta geológica do Brasil ao milionésimo MME-DNPM. Folha São Francisco (SG-23): 57 pp.

CALDASSO, A. L., ENNES, E. R. et GRAZIA, C. A. - 1973 - Projeto Sudeste do Piauí. DNPM/ CPRM, relatório final integrado

CERVELLE, B. - 1967 - Contribution à 1 étude de la série ilménite-geikielite. Bull. BRGM 6: 1-26

CHEVALliER, R., BOLFA, J. et MATHIEU, S. - 1955 - Titanomagnétites et ilménites ferromagnétiques. I: Étude optique, radiocristallographique et chimique. Bull. Soc. franç. Min. Cristall. 78: 307-346

HAMMOND, P. - 1952 - Allard Lake ilmenite deposits. Econ. Geol. 47 (6): 634-649

LOMBARD, J. - 1957 - Sur la géochimie et les gisements de titane. A la recherche du rutile. Etude bibliographique. Chronique des Mines d'Outremer et de la Rech. Min. 25: (253/254): 234-263

ROSE, E. R. - 1969 - Geology of titanium and titaniferous deposits of Canada. Geol. Survey of Canada Depart. of Energy, Mines and Resources. Econ. Geol. Report 25: 177 pp.

ROUTHIER, P. - 1963 - Les gisements métallifères. Géologie et principes de recherches. 1282 pp.

WINGE, M. - 1968 - Geologia da região das Serras do Estreito e Boqueirão. NW da Bahia e SSE do Piauí. Sudene D.R.N.-Div. Geol. Série Geol. Reg. 17 